Proceeding Series of the Brazilian Society of Computational and Applied Mathematics

\title{
Solução da partícula livre em 3D através dos métodos de fatorização e supersimetria
}

\author{
Gisele Bosso de Freitas ${ }^{1}$ \\ Departamento de Bioestatística, IBB/UNESP, Botucatu, SP \\ Elso Drigo Filho ${ }^{2}$ \\ Departamento de Física, IBILCE/UNESP, São José do Rio Preto, SP
}

Resumo. Neste trabalho são utilizados os métodos de fatorização e supersimetria em mecânica quântica para estudar a partícula livre tridimensional. Através desse estudo, notamos que há a possibilidade da construção da hierarquia de hamiltonianos e com isso a solução através dos métodos mais comumente apresentados na literatura é recuperada.

Palavras-chave. fatorização, supersimetria, poço de potencial tridimencional.

\section{Introdução}

Poços de potenciais podem ser utilizados para descrever desde moléculas biológicas até estruturas eletrônicas, como exemplo podemos citar modelos de estruturas semicondutoras, geralmente contruídas por arsenídio de gálio (GaAs) [1].

Tradicionalmente em livros de mecânica quântica [3] o estudo da partícula livre 3D é tratado resolvendo-se diretamente a equação de Schrödinger, ou seja, determinando as autofunções e autovalores através da resolução direta dessa equação diferencial de segunda ordem. No presente trabalho é mostrado o uso dos métodos de fatorização e supersimetria para a solução da equação diferencial que descreve esse sistema. Como esperado, os resultados são análogos àqueles obtidos por outros métodos.

O formalismo resultante da Mecânica Quântica Supersimétrica [2], tem sido usado com muito sucesso na análise tanto de problemas quânticos quanto de problemas biológicos [10]; no tratamento de potenciais exatamente, [15], e parcialmente solúveis, [4].

\section{Métodos de fatorização e supersimetria em mecânica quântica}

Uma maneira geral de escrever o hamiltoniano da equação de Shrödinger é

$$
H \psi(x)=\epsilon \psi(x) .
$$

\footnotetext{
${ }^{1}$ freitas.gibb@gmail.com

2elso@ibilce.unesp.br
} 
Quando o método de fatorização é utilizado para obter as soluções da equação de Schrödinger, o hamiltoniano é substituído pela combinação de dois operadores diferenciais de primeira ordem. Então, dado um operador diferencial de segunda ordem, o objetivo do método é encontrar dois operadores [11,13]

$$
A=\frac{d}{d x}+\beta(x) \quad e \quad A^{\dagger}=-\frac{d}{d x}+\beta(x),
$$

de modo que o hamiltoniano possa ser escrito como

$$
H=\frac{d^{2}}{d x^{2}}+V(x)=A^{\dagger} A+\epsilon,
$$

em que $\epsilon$ e conhecida como energia de fatorização (comparável à energia do sistema). Substituindo (1) em (2), a equação de Ricatti é

$$
\beta^{2}(x)-\beta^{\prime}(x)+\epsilon=V(x),
$$

em que $\beta^{\prime}(x)$ é a derivada de primeira ordem da função $\beta(x)$. Uma vez encontrada a solução para a equação (3), a função $\beta(x)$ leva às funções de onda através de

$$
\psi(x) \propto e^{-\int^{x} \beta(y) d y} .
$$

Quando se utiliza o formalismo da supersimetria em mecânica quântica [14] para estudar sistemas descritos pela equação de Schrödinger, os dois operadores de primeira ordem, citados anteriormente, são conhecidos como operadores bosônicos escritos em termos do superpotencial $w_{1}(x)$ da forma

$$
a_{1}=\frac{d}{d x}+w_{1}(x) \quad e \quad a_{1}^{\dagger}=-\frac{d}{d x}+w_{1}(x) .
$$

O hamiltoniano supersimétrico $H_{S S}$ é dado por

$$
H_{s s}=\left(\begin{array}{cc}
a_{1}^{\dagger} a_{1} & 0 \\
0 & a_{1} a_{1}^{\dagger}
\end{array}\right)=\left(\begin{array}{cc}
H_{1} & 0 \\
0 & H_{2}
\end{array}\right)
$$

em que $H_{1}$ e $H_{2}$ são hamiltonianos companheiros supersimétricos. Há uma relação direta entre esses hamiltonianos e suas autofunções e autovalores, dada por,

$$
\begin{array}{r}
\psi_{n+1}^{(1)}(x) \propto a_{1}^{\dagger} \psi_{n}^{(2)}(x), \psi_{n}^{(2)}(x) \propto a_{1} \psi_{n+1}^{(1)}(x) \\
E_{n+1}^{(1)}=E_{n}^{(2)}
\end{array}
$$

$\operatorname{com} E_{0}^{(1)}=0,(n=0,1, \ldots)$.

A partir de (6) tem-se a equação de Riccati,

$$
w^{2}(x)-w^{\prime}(x)+E_{0}^{(1)}=V(x),
$$


Este processo pode ser repetido $n$ vezes, criando uma hierarquia de hamiltonianos, escritos de forma geral como,

$$
H_{n}=a_{n}^{\dagger} a_{n}
$$

com

$$
a_{n}=\frac{d}{d x}+w_{n}(x) \quad e \quad a_{n}^{\dagger}=-\frac{d}{d x}+w_{n}(x) .
$$

A supersimetria permite relacionar todos os $n$ membros da hierarquia, de acordo com

$$
\begin{array}{r}
\psi_{n}^{(1)}(x) \propto a_{1}^{\dagger} a_{2}^{\dagger} \ldots a_{n}^{\dagger} \psi_{0}^{(n+1)}(x), \\
E_{n}^{(1)}=E_{0}^{(n+1)}
\end{array}
$$

em que as autofunções $\psi_{n}^{(1)}(x)$ são normalizáveis.

\section{Partícula livre tridimensional}

O potencial do poço quadrado infinito tridimensional, que descreve uma partícula livre, pode ser escrito matematicamente como

$$
V(r)=\left\{\begin{array}{cl}
0, & \text { if } 0 \leq r \leq L \\
\infty, & \text { if } \quad r>L
\end{array}\right.
$$

Para a partícula sob a ação desse potencial (14) a equação de Schrödinger radial [3], fazendo por simplicidade $\hbar=2 \mu=1$ e introduzindo a função $\phi(r)$ de acordo com: $R(r)=\frac{\phi(r)}{r}$. Como de costume $l$ é o número quântico de momento angular. Tem-se

$$
-\left[\frac{d^{2}}{d r^{2}}+\frac{2}{r} \frac{d}{d r}-\frac{l(l+1)}{r^{2}}\right] \frac{\phi(r)}{r}-\epsilon \frac{\phi(r)}{r}=0 .
$$

\subsection{Solução Via Método de Fatorização}

De maneira geral, a fatorização do hamiltoniano da Eq.(15) resulta na seguinte equação de Ricatti

$$
\beta_{n, l}^{2}(r)-\beta_{n, l}^{\prime}(r)+\epsilon_{n, l}=\frac{l(l+1)}{r^{2}} .
$$

As soluções aqui obtidas, são as funções conhecidas como funções eféricas de Bessel $\left(j_{l}(b r)\right)$ dividas por $r$. Assim temos os superpotenciais

$$
\beta_{n, l}(r)=-b_{n, l} \frac{j_{n, l+1}\left(b_{n, l} r\right)}{j_{n, l}\left(b_{n, l} r\right)},
$$

com autovalores de energia dados por

$$
\epsilon_{n, l}=b_{n, l}^{2}
$$

e autofunções da forma

$$
\phi_{n, l}(r)=N \frac{j_{n, l}\left(b_{n, l} r\right)}{r}
$$




\subsection{Solução Via Supersimetria em Mecânica Quântica}

A Eq. (16) pode ser resolvida para diferentes valores de $l$. Para $l=0$, matematicamente, retorna-se ao problema do poço de potencial quadarado infinito apresentado na seção II. Para $l=1$ obtém-se como superpotencial a função

$$
w_{1}(r)=\frac{1}{r}-\frac{b^{2} r \sin (b r)}{\sin (b r)-b r \cos b r},
$$

como o autovalor de energia

$$
E_{0}^{(1)}=b^{2}
$$

e como autofunção para este estado

$$
R(r)=\frac{\sin (b r)}{b^{2} r}-\frac{\cos (b r)}{b}
$$

Para encontrar o valor de $b$, basta aplicar as condições de contorno na autofunção (22), ou seja,

$$
\begin{aligned}
& \lim _{r \rightarrow 0} R(r)=0 ; \\
& \lim _{r \rightarrow L} R(r)=0 .
\end{aligned}
$$

Este procedimento conduz à seguinte equação transcendental

$$
b L \cos (b L)=\sin (b L) .
$$

As soluções para Eq. (25) podem ser obtidas através de métodos gráficos, para um exemplo numérico, pode-se usar $L=1$, então os dois primeiros valores para $b$ são 4.493 e 7.725. Como este valor refere-se ao estado de mais baixa energia e $b$ está diretamente relacionado com a energia deste estado, escolhe-se o menor valor para esta constante.

Seguindo no procedimendo usual $[2,8]$, encontramos a hierarquia de hamiltonianos, ou seja, para $l \geq 0$, as soluções com $n=0,1, \ldots$, são dadas por

$$
w_{n+1, l}(r)=-\frac{\bar{a}_{1} \ldots \bar{a}_{n} \frac{j_{n, l}\left(b_{n, l} r\right)}{r}}{a_{1} \ldots a_{n} \frac{j_{n, l}\left(b_{n, l} r\right)}{r}},
$$

em que $a_{n}=\frac{d}{d r}+w_{n}(r)$ são os operadores bosônicos e $\bar{a}_{n}=\frac{d^{2}}{d r^{2}}+\frac{d}{d r} w_{n}(r)$. Os autovalores de energia são dados por

$$
E_{n-1, l}^{(1)}=b_{n, l}^{2},
$$

e as autofunções tem a forma

$$
\phi_{n-1, l}(r)=N a_{1} \ldots a_{n} \frac{j_{n, l}\left(b_{n, l} r\right)}{r} .
$$

Em que os valores de $b_{n, l}$ são determinados através da aplicação das condições de contorno na Eq. (28). As soluções são encontradas através de métodos gráficos e algumas soluções estão dispostas na tabela (1), com $n=1,2, \ldots$ correspondendo às soluções aceitáveis.

Para o problema original, as autofunções são

$$
\phi_{n, l}(r)=N \frac{j_{n, l}\left(b_{n, l} r\right)}{r} .
$$


Tabela 1: Valores das raízes das funções esféricas de Bessel.

\begin{tabular}{|c|c|}
\hline Estado & $b_{n, l}$ \\
\hline $1 \mathrm{~s}$ & 3.142 \\
\hline $1 \mathrm{p}$ & 4.493 \\
\hline $1 \mathrm{~d}$ & 5.763 \\
\hline $2 \mathrm{~s}$ & 6.283 \\
\hline $1 \mathrm{f}$ & 6.988 \\
\hline $2 \mathrm{p}$ & 7.725 \\
\hline
\end{tabular}

\section{Conclusão}

Neste trabalho, utilizou-se os métodos de fatorização e de supersimetria em mecânica quântica para estudar a equação de Schrödinger para a partícula livre. Os resultados obtidos com este estudo mostraram correspondência com os encontrados na literatura [3].

Os resultados apresentados na tabela (1) expressam números quânticos (valores que definem a quantidade de energia do elétron) para alguns subníveis de energia. É através desses números que é possível caracterizar um átomo.

Apesar da simplicidade dos métodos utilizados neste trabalho, eles se mostram bastante úteis, tendo sido aplicadas em vários contextos com êxito. Através dos resultados obtidos, vemos que a abordagem proposta pode ser proveitosamente usada para o estudo de problemas envolvendo sistemas tridimensionais.

\section{Agradecimentos}

Os autores agradecem à UNESP e à CAPES pelo apoio financeiro parcial.

\section{Referências}

[1] Y. Ando and A. Cappy, Ensemble Monte Carlo simulation for electron transport in quantum wire structures, J. App. Phys., vol. 74, 3983-3992, (1993).

[2] F. Cooper, A. Khare and U.P. Sukhatme, Supersymmetry in Quantum Mechanics, Singapore, World Scientific, (2001).

[3] A.S. Davidov, Quantum Mechanics, Mass, Addison-Wesley, (1968).

[4] E. Drigo Filho e R.M. Ricotta, Supersymmetric Quantum Mechanics and Partially Solvable Potential, Phys. At. Nucl., vol. 61, 1722-1725, (1998).

[5] E. Drigo and Ricotta R.M., Ladder operators for subtle hidden shape-invariant potentials, J. Phys. A: Math. Gen., vol. 37, 10057-10064, (2004).

[6] R. Eisberg and R. Resnick, Quantum physics, London, John Wiley \& Sons, (1974). 
[7] S. Gasiorowicz, Quantum Physics, New York, John Wiley, (1996).

[8] G. Junker, Supersymetric Methods in Quantum and Statistical Physics, Berlin, Springer, (1996).

[9] A. Khare and U.P. Sukhatme, Complex periodic potentials with a finite number of band gaps, J. Math. Phys., vol. 47, 062103-1-062103-22, (2006).

[10] F. Polotto, M. T. Araujo, E. Drigo Filho, Solutions of the Fokker Planck equation for a Morse isospectral potential, J. Phys. A, Math. Theo., vol. 43, 015207-1-015207-9, (2010).

[11] J. O. Rosas-Ortiz, On the factorization method in quantum mechanics, (A. Ballestrero, et al, eds.), 285-299, Burgos, Spain, (1999).

[12] L.I. Schiff, Quantum Mechanics, New York, McGraw-Hill, (1968).

[13] E. Schrödinger, A method of determining quantum-mechanical eigenvalues and eigenfunctions, Proc. Roy. Irish Acad. A, vol. 46, 9-16, (1940).

[14] G. Stedman, Simple supersymmetry: II. Factorisation method in quantum mechanics, Eur. J. Phys. vol. 6, 225-231 (1985).

[15] C.V. Sukumar, Supersymmetry, factorisation of the Schrödinger equation and a Hamiltonian hierarchy, J. Phys. A: Math. Gen., vol.18, L57-L61 (1985).

[16] E. Witten, Dynamical breaking of supersymmetry, Nucl. Phys. B, vol. 188, 513-554, (1981). 